\title{
Blind Direction-of-Arrival Estimation with Uniform Circular Array in Presence of Mutual Coupling
}

\author{
Song Liu, Lisheng Yang, Shizhong Yang, Qingping Jiang, and Haowei Wu \\ Key Laboratory of Aerocraft Tracking Telemetering \& Command and Communication, Ministry of Education, Chongqing University, \\ Chongqing 400030, China \\ Correspondence should be addressed to Lisheng Yang; yls@cqu.edu.cn
}

Received 3 November 2015; Revised 11 December 2015; Accepted 15 December 2015

Academic Editor: Shih Yuan Chen

Copyright (C) 2016 Song Liu et al. This is an open access article distributed under the Creative Commons Attribution License, which permits unrestricted use, distribution, and reproduction in any medium, provided the original work is properly cited.

\begin{abstract}
A blind direction-of-arrival (DOA) estimation algorithm based on the estimation of signal parameters via rotational invariance techniques (ESPRIT) is proposed for a uniform circular array (UCA) when strong electromagnetic mutual coupling is present. First, an updated UCA model with mutual coupling in a discrete Fourier transform (DFT) beam space is deduced, and the new manifold matrix is equal to the product of a centrosymmetric diagonal matrix and a Vandermonde-structure matrix. Then we carry out blind DOA estimation through a modified ESPRIT method, thus avoiding the need for spatial angular searching. In addition, two mutual coupling parameter estimation methods are presented after the DOAs have been estimated. Simulation results show that the new algorithm is reliable and effective especially for closely spaced signals.
\end{abstract}

\section{Introduction}

Uniform circular array based DOA estimation methods are always attractive because the UCA has a special symmetric structure that provides almost the same resolution ability along the $360^{\circ}$ azimuth angle domain. Except for conventional DOA estimation methods such as beam space searching or the Capon method [1], many other methods can be applied, such as the commonly used multiple signal classification (MUSIC) [2] or the well-known ESPRIT [3] in phase mode space. More effective methods such as UCA-RBMUSIC and UCA-ESPRIT [4] have been introduced, and the mapping error reducing method was developed [5].

However, the electromagnetic mutual coupling effect cannot be ignored in a real array. Generally, this effect will severely degrade the performance of the above methods [6]. A classic DOA estimation method based on an iterative search technique was proposed to estimate the DOA and the mutual coupling matrix (MCM) parameters jointly [7], but it has a high computation cost. The rank reduction (RARE) method introduced by [7] was further developed to obtain the DOA estimate for a UCA [8-10], but an angular search is still needed. Moreover, angular ambiguities are challenges for
RARE-based blind methods. For example, two closely spaced signals cannot be differentiated according to the UCA-RARE [8] spectrum because there is a spurious peak at the middle of the two true angles.

In this paper, we design a new modified ESPRIT method for UCA to estimate the azimuth angle when mutual coupling is present. The proposed method is blind to mutual coupling and can completely avoid angular searching. Reliable DOA estimates can be obtained, especially for closely spaced signals. In addition, we will introduce two methods to estimate the MCM parameters once the DOA values are calculated.

\section{UCA Model with Mutual Coupling}

Suppose $M$-element UCA has a radius $r$ (Figure 1). All of the antenna elements are identical, and there are $D$ far-field narrow signals impinging from $\left\{\theta_{j}, j=1, \ldots, D\right\}$ which are the parameters to be estimated. The snapshot can be written as

$$
\mathbf{z}(k)=\mathbf{C A s}(k)+\mathbf{n}(k), \quad k=1, \ldots, K .
$$




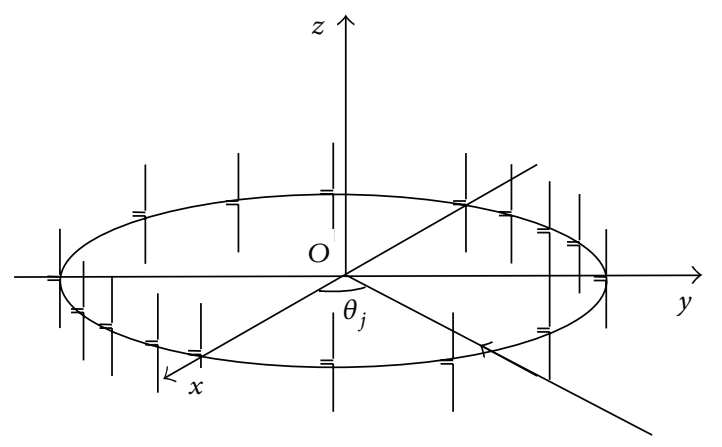

FIgURE 1: Uniform circular array.

$K$ is the total sampling number and $\mathbf{A}$ is the manifold matrix:

$$
\begin{aligned}
\mathbf{A} & =\left[\mathbf{a}\left(\theta_{1}\right), \ldots, \mathbf{a}\left(\theta_{D}\right)\right], \\
\left(\mathbf{a}\left(\theta_{j}\right)\right)_{m} & \\
& =f\left(\theta_{j}\right) \exp \left(i \frac{2 \pi r}{\lambda} \cos \left(\theta_{j}-\frac{2(m-1) \pi}{M}\right)\right),
\end{aligned}
$$

where $m=1, \ldots, M . \lambda$ is the wavelength of the signals, and $\mathbf{a}\left(\theta_{j}\right)$ is the manifold vector for the $j$ th signal. $f\left(\theta_{j}\right)$ is the common directivity factor of the antenna elements. Since all the antenna elements are identical, $f\left(\theta_{j}\right)$ can be normalized as 1 , and we use effective SNR which has already included the directivity factor. $\mathbf{s}(k)$ is the signal sample vector:

$$
\mathbf{s}(k)=\left[s_{1}(k), \ldots, s_{D}(k)\right]^{T} .
$$

C is the MCM, which is a symmetric circular Toeplitz matrix with the form

$$
\mathbf{C}=\left[\begin{array}{cccccc}
c_{0} & c_{1} & \ldots & c_{N} & \ldots & c_{1} \\
c_{1} & c_{0} & \ldots & c_{N-1} & \ldots & c_{2} \\
\vdots & \vdots & \ldots & \vdots & \ldots & \vdots \\
c_{N} & c_{N-1} & \ldots & c_{0} & \ldots & c_{N-1} \\
\vdots & \vdots & \ldots & \vdots & \ldots & \vdots \\
c_{1} & c_{2} & \ldots & c_{N-1} & \ldots & c_{0}
\end{array}\right], \quad N=\left\lfloor\frac{M}{2}\right\rfloor .
$$

$\lfloor\cdot\rfloor$ is the flooring function and $c_{0}$ is normalized as 1 . For ideal dipole antenna array (Figure 1), induced EMF (Electromotive Force) method can give a close form of self-impedance and mutual impedance and thus MCM can be calculated according to Gupta and Ksienski's formulation [6].

Suppose the signals are uncorrelated and $\mathbf{n}(k)$ is white Gaussian noise:

$$
\begin{aligned}
E[\mathbf{s}(k)] & =\mathbf{0}, \\
E\left[\mathbf{s}(k) \mathbf{s}(k)^{H}\right] & =\mathbf{R}_{s}, \\
E[\mathbf{n}(k)] & =\mathbf{0}, \\
E\left[\mathbf{n}(k) \mathbf{n}(k)^{H}\right] & =\sigma_{w}^{2} \mathbf{I} .
\end{aligned}
$$

\section{Algorithm}

3.1. UCA Model in DFT Beam Space. First we introduce the DFT of the MCM for UCA.

Lemma 1 (see [11-13]). If $\mathbf{C}$ is a circular matrix with its first column vector as $\mathbf{c}=\left[c_{0}, c_{1}, \ldots, c_{M-1}\right]^{T}$ and $\mathbf{F}$ is a Fourier matrix

$$
\begin{aligned}
\mathbf{F} & =\left[\mathbf{w}_{0}, \mathbf{w}_{1}, \ldots, \mathbf{w}_{M-1}\right], \\
\mathbf{w}_{j} & \\
& =\frac{1}{\sqrt{M}}\left[1, \exp \left(i \frac{2 \pi j}{M}\right), \ldots, \exp \left(i \frac{2 \pi j(M-1)}{M}\right)\right]^{T},
\end{aligned}
$$

where $j=0, \ldots, M-1$, then $\mathbf{\Lambda}=\mathbf{F} \mathbf{C F}^{H}$ is a diagonal matrix with entries as $\mathbf{C}$ 's eigenvalues:

$$
\mu_{m}=(\Lambda)_{(m+1)(m+1)}=\sum_{j=0}^{M-1} c_{j} \exp \left(i \frac{2 \pi j m}{M}\right)
$$

in which $m=0,1, \ldots, M-1$. For the Fourier matrix $\mathbf{F}$

$$
\begin{aligned}
\mathbf{F} & =\mathbf{F}^{T}, \\
\mathbf{F}^{H} & =\mathbf{F}^{*} .
\end{aligned}
$$

If $\mathbf{C}$ is also symmetric, this means that

$$
\begin{aligned}
\mathbf{C} & =\mathbf{C}^{T} \\
\text { or } c_{j} & =c_{M-j}, \quad j=1, \ldots, M-1 .
\end{aligned}
$$

Then we can rewrite $\Lambda$ as

$$
\begin{aligned}
\mu_{m} & =\mathbf{w}_{m}{ }^{T} \mathbf{C} \mathbf{w}_{m}{ }^{*}=\left(\mathbf{w}_{m}{ }^{T} \mathbf{C} \mathbf{w}_{m}{ }^{*}\right)^{T}=\mathbf{w}_{m}{ }^{H} \mathbf{C} \mathbf{w}_{m}, \\
0 & =\mathbf{w}_{m}{ }^{T}{ }^{T} \mathbf{w}_{j}{ }^{*} \quad m \neq j
\end{aligned}
$$

and we have

$$
\mu_{M-m}=\left(\mathbf{w}_{M-m}\right)^{H} \mathbf{C}\left(\mathbf{w}_{M-m}\right)=\mathbf{w}_{m}{ }^{T} \mathbf{C} \mathbf{w}_{m}{ }^{*}=\mu_{m}
$$

where $m=1, \ldots, M-1$. From (8) and (12), we get a linear equation which $\mathbf{c}^{\prime}$ and $\boldsymbol{\mu}^{\prime}$ should satisfy for even $M$ :

$$
\Theta c^{\prime}=\mu^{\prime}
$$

where 


$$
\begin{aligned}
\mathbf{c}^{\prime}: & =\left[c_{0}, \ldots, c_{M / 2}\right]^{T}, \\
\boldsymbol{\mu}^{\prime}:= & {\left[\mu_{0}, \ldots, \mu_{M / 2}\right]^{T}, } \\
\Theta: & {\left[\begin{array}{ccccc}
1 & \ldots & 2 & 1 \\
1 & 2 \cos \left(\frac{2 \pi}{M} \cdot 1 \cdot 1\right) & \ldots & 2 \cos \left(\frac{2 \pi}{M} \cdot 1 \cdot \frac{M-2}{2}\right) & -1 \\
1 & 2 \cos \left(\frac{2 \pi}{M} \cdot 2 \cdot 1\right) & \ldots & 2 \cos \left(\frac{2 \pi}{M} \cdot 2 \cdot \frac{M-2}{2}\right) & 1 \\
\vdots & \vdots & \ldots & \vdots \\
1 & 2 \cos \left(\frac{2 \pi}{M} \cdot \frac{M-2}{2} \cdot 1\right) & \ldots & 2 \cos \left(\frac{2 \pi}{M} \cdot \frac{M-2}{2} \cdot \frac{M-2}{2}\right) & -1 \\
1 & 2 \cos \left(\frac{2 \pi}{M} \cdot \frac{M}{2} \cdot 1\right) & \cdots & 2 \cos \left(\frac{2 \pi}{M} \cdot \frac{M}{2} \cdot \frac{M-2}{2}\right) & 1
\end{array}\right] . }
\end{aligned}
$$

Therefore, if we determine the estimate of $\boldsymbol{\mu}^{\prime}$, then we can obtain the estimate of the mutual coupling parameters $c^{\prime}$ by (13). In addition, there are similar equations where $M$ is odd.

According to [4], we set

$$
\begin{aligned}
& \beta=\frac{2 \pi r}{\lambda}, \\
& L=\lfloor\beta\rfloor,
\end{aligned}
$$

where $L$ is the number of excited phase modes. We define another Fourier matrix $\mathbf{F}^{\prime}$, which is different from (6):

$$
\begin{aligned}
& \mathbf{F}^{\prime}=\left[\mathbf{F}_{1}, \mathbf{F}_{2}\right], \\
& \mathbf{F}_{1}=\left[\mathbf{w}_{-L}, \ldots, \mathbf{w}_{L}\right], \\
& \mathbf{F}_{2}=\left[\mathbf{w}_{L+1}, \ldots, \mathbf{w}_{M-L-1}\right] .
\end{aligned}
$$

Then the snapshot in DFT beam space is

$$
\widetilde{\mathbf{z}}(k)=\mathbf{F}^{\prime H} \mathbf{z}(k)=\mathbf{F}^{\prime H} \mathbf{C F}^{\prime} \mathbf{F}^{\prime H} \mathbf{A s}(k)+{\mathbf{F}^{\prime}}^{H} \mathbf{n}(k),
$$

if we write $\mathbf{F}^{\prime H} \mathbf{C F}^{\prime}$ as

$$
\mathbf{F}^{\prime H} \mathbf{C F}^{\prime}=\left[\begin{array}{cc}
\boldsymbol{\Gamma}_{C} & \mathbf{0} \\
\mathbf{0} & \boldsymbol{\Gamma}_{C}^{\prime}
\end{array}\right],
$$

where (see (11), (12), and (18))

$$
\Gamma_{C}=\operatorname{diag}\left(\left[\mu_{L}, \ldots, \mu_{1}, \mu_{0}, \mu_{1}, \ldots, \mu_{L}\right]\right) .
$$

In addition, we can obtain [4]

$$
\mathbf{F}^{\prime H} \mathbf{A}=\left[\begin{array}{l}
\mathbf{F}_{1}{ }^{H} \mathbf{A} \\
\mathbf{F}_{2}{ }^{H} \mathbf{A}
\end{array}\right]=\left[\begin{array}{c}
\boldsymbol{\Gamma}_{J} \tilde{\mathbf{A}} \\
\mathbf{F}_{2}{ }^{H} \mathbf{A}
\end{array}\right]
$$

with

$$
\boldsymbol{\Gamma}_{J}=\sqrt{M} \operatorname{diag}\left(\left[i^{-L} J_{-L}(\beta), \ldots, i^{L} J_{L}(\beta)\right]\right) .
$$

$J_{l}(\cdot)$ is $l$ order first-kind Bessel function. According to its property

$$
J_{-l}(\beta)=(-1)^{l} J_{l}(\beta)
$$

we get

$$
\Gamma_{J}=\sqrt{M} \operatorname{diag}\left(\left[\alpha_{L}, \alpha_{L-1}, \ldots, \alpha_{0}, \ldots, \alpha_{L-1}, \alpha_{L}\right]\right),
$$

where

$$
\alpha_{l}=i^{l} J_{l}(\beta), \quad l=0,1, \ldots, L
$$

$\widetilde{\mathbf{A}}$ is the updated manifold matrix with a Vandermonde structure

$$
\widetilde{\mathbf{A}}=\left[\begin{array}{ccc}
\exp \left(-i L \theta_{1}\right) & \cdots & \exp \left(-i L \theta_{D}\right) \\
\vdots & \cdots & \vdots \\
\exp \left(i L \theta_{1}\right) & \cdots & \exp \left(i L \theta_{D}\right)
\end{array}\right]
$$

Finally, we obtain the snapshot in DFT beam space

$$
\widetilde{\mathbf{z}}(k)=\left[\begin{array}{c}
\Gamma \widetilde{\mathbf{A}} \\
\Gamma_{C}^{\prime} \mathbf{F}_{2}{ }^{H} \mathbf{A}
\end{array}\right] \mathbf{s}(k)+\widetilde{\mathbf{n}}(k)
$$

in which

$$
\begin{aligned}
\boldsymbol{\Gamma} & =\boldsymbol{\Gamma}_{C} \boldsymbol{\Gamma}_{J}, \\
E\left[\widetilde{\mathbf{n}}(k) \widetilde{\mathbf{n}}(k)^{H}\right] & =\sigma_{w}^{2} \mathbf{I} .
\end{aligned}
$$

3.2. DOA Estimation and MCM Calculation. We carry out the eigendecomposition on the covariance matrix $\mathbf{R}_{\widetilde{z}}$ and obtain the signal subspace $\widetilde{\mathbf{U}}_{s}$, which consists of eigenvectors corresponding to $D$ maximum eigenvalues. Select the first $L+1$ rows of $\widetilde{\mathbf{U}}_{s}$ as $\widetilde{\mathbf{U}}_{s 1}$ and the second $L+1$ rows as $\widetilde{\mathbf{U}}_{s 2}$. Select the first $L+1$ rows of $\widetilde{\mathbf{A}}$ as $\widetilde{\mathbf{A}}_{1}$ and the second $L+1$ rows as $\widetilde{\mathbf{A}}_{2}$. Select the first $(L+1) \times(L+1)$ diagonal matrix of $\boldsymbol{\Gamma}$ as $\boldsymbol{\Gamma}_{1}$ and the second $(L+1) \times(L+1)$ diagonal matrix as $\boldsymbol{\Gamma}_{2}$. Use 
the same notations for other matrices $\Gamma_{J 1}, \Gamma_{J 2}, \Gamma_{C 1}$, and $\Gamma_{C 2}$. Then we have

$$
\begin{aligned}
& \widetilde{\mathbf{U}}_{s 1}=\Gamma_{1} \widetilde{\mathbf{A}}_{1} \mathbf{T}, \\
& \widetilde{\mathbf{U}}_{s 2}=\Gamma_{2} \widetilde{\mathbf{A}}_{2} \mathbf{T},
\end{aligned}
$$

where $\mathbf{T}$ is a nonsingular matrix. Thus we get

$$
\Gamma_{12} \widetilde{U}_{s 2}=\widetilde{\mathbf{U}}_{s 1} \Psi
$$

with

$$
\begin{aligned}
\boldsymbol{\Gamma}_{12} & =\boldsymbol{\Gamma}_{1} \boldsymbol{\Gamma}_{2}^{-1}=\boldsymbol{\Gamma}_{J 1} \boldsymbol{\Gamma}_{C 1} \boldsymbol{\Gamma}_{J 2}{ }^{-1} \boldsymbol{\Gamma}_{C 2}{ }^{-1}=\operatorname{diag}(\mathbf{d}), \\
\mathbf{d} & =\left[\frac{\mu_{L} \alpha_{L}}{\mu_{L-1} \alpha_{L-1}}, \frac{\mu_{L-1} \alpha_{L-1}}{\mu_{L-2} \alpha_{L-2}}, \ldots, \frac{\mu_{1} \alpha_{1}}{\mu_{0} \alpha_{0}}, \frac{\mu_{0} \alpha_{0}}{\mu_{1} \alpha_{1}}\right]^{T}, \\
\Psi & =\mathbf{T}^{-1} \operatorname{diag}\left(\left[\exp \left(i \theta_{1}\right), \ldots, \exp \left(i \theta_{D}\right)\right]\right) \mathbf{T} .
\end{aligned}
$$

Since we use sampled data, we should replace $\widetilde{\mathbf{U}}_{s}$ with $\widehat{\widetilde{\mathbf{U}}}_{s}$ and define the object function as

$$
\begin{array}{cl}
\{\widehat{\mathbf{d}}, \widehat{\Psi}\}=\underset{\mathbf{d}, \Psi}{\arg \min } & \left\|\operatorname{diag}(\mathbf{d}) \widehat{\widetilde{\mathbf{U}}}_{s 2}-\widehat{\widetilde{\mathbf{U}}}_{s 1} \Psi\right\| \\
\text { s.t. } & \widehat{\mathbf{d}}_{L} \widehat{\mathbf{d}}_{L+1}=1 .
\end{array}
$$

We use the solution to the above equations from $[14,15]$. Consider

$$
\begin{aligned}
\widehat{\mathbf{d}}=\underset{\mathbf{d}}{\arg \min } & \mathbf{d}^{H} \mathbf{Q d} \\
\text { s.t. } & \widehat{\mathbf{d}}_{L} \widehat{\mathbf{d}}_{L+1}=1 \\
\widehat{\Psi} & =\left(\widehat{\widetilde{\mathbf{U}}}_{s 1}{ }^{H} \widehat{\widetilde{\mathbf{U}}}_{s 1}\right)^{-1} \widehat{\widetilde{\mathbf{U}}}_{s 1}^{H} \operatorname{diag}(\widehat{\mathbf{d}}) \widehat{\widetilde{\mathbf{U}}}_{s 2}
\end{aligned}
$$

with

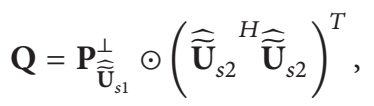

where $\mathbf{P}_{\widehat{\widetilde{\mathbf{U}}}_{s 1}^{\perp}}^{\perp} \mathbf{I}-\widehat{\widetilde{\mathbf{U}}}_{s 1}\left(\widehat{\widetilde{\mathbf{U}}}_{s 1}{ }^{H} \widehat{\widetilde{\mathbf{U}}}_{s 1}\right)^{-1} \widehat{\widetilde{\mathbf{U}}}_{s 1}{ }^{H}$ and $\odot$ is the Hadamard product. If $\boldsymbol{\psi}_{\text {min }}$ is the eigenvector of $\mathbf{Q}$ corresponding to its minimum eigenvalue, then we have

$$
\begin{aligned}
\widehat{\mathbf{d}} & =\frac{\psi_{\min }}{\rho} \\
\rho^{2} & =\left(\psi_{\min }\right)_{L}\left(\psi_{\min }\right)_{L+1} .
\end{aligned}
$$

Equation (37) indicates that there is a phase $\pi$ ambiguity for $\rho$, and thus it will introduce a $\pi$ ambiguity to the DOA values through the eigenvalues of $\widehat{\Psi}$. However, this ambiguity can be cleared by comparing the RARE spectrum [9] values on $\widehat{\theta}_{j}$ and $\widehat{\theta}_{j}+\pi$. We mark the estimated vector as $\widehat{\mathbf{d}}_{\text {opt }}$ without $\pi$ ambiguity.

Finally, we can determine the DOA estimates through the eigenvalues of $\widehat{\boldsymbol{\Psi}}$. Following is the detailed procedure of the blind method for DOA estimation of $\left\{\theta_{j}\right\}$ :

(1) Calculate the sample covariance matrix $\widehat{\mathbf{R}}_{z}$ and do eigendecomposition. Get the estimated signal subspace $\widehat{\mathbf{U}}_{s}$ and noise subspace $\widehat{\mathbf{U}}_{n}$.

(2) Get the updated signal subspace $\widehat{\widetilde{U}}_{s}$. Select the first $L+$ 1 rows as $\widehat{\widetilde{U}}_{s 1}$ and the second $L+1$ rows as $\widehat{\widetilde{U}}_{s 2}$ :

$$
\widehat{\widetilde{\mathbf{U}}}_{s}=\mathbf{F}^{\prime H} \widehat{\mathbf{U}}_{s}
$$

(3) Estimate the vector $\widehat{\mathbf{d}}$ and fitting matrix $\widehat{\boldsymbol{\Psi}}$ according to (34)-(36). Carry out eigendecomposition on $\widehat{\Psi}$ and get the DOA estimates $\left\{\widehat{\theta}_{j}\right\}$.

(4) Compare the blind RARE spectral values [9] on $\hat{\theta}_{j}$ and $\widehat{\theta}_{j}+\pi$ and clear the ambiguity. Output the final DOA estimates.

We label the above mentioned method as "Blind-ml-half" because we only select $L+1$ rows of $\widehat{\widehat{U}}_{s}$. In addition, we can select the first $2 L$ and the second $2 L$ rows from $\widehat{\widehat{U}}_{s}$ as $\widehat{\widetilde{U}}_{s 1}$ and $\widehat{\widehat{U}}_{s 2}$. This method is labeled "Blind-m1-full." Furthermore, we can select the first $L$ and the third $L$ rows from $\widehat{\widehat{U}}_{s}$ as $\widehat{\widetilde{U}}_{s 1}$ and $\widehat{\widetilde{U}}_{s 2}$ or select the first $2 L-1$ and the third $2 L-1$ rows from $\widehat{\widetilde{U}}_{s}$ as $\widehat{\widetilde{U}}_{s 1}$ and $\widehat{\widetilde{U}}_{s 2}$. We label the two methods as "Blind$\mathrm{m} 2$-half" and "Blind-m2-full," respectively. We will carry out simulations on these four methods in Section 4. $\widehat{\theta}$.

Now we can estimate the MCM parameters once we get

Method 1. If $c_{j}=0, j>L$, then, according to (32) and (13), we get

$$
\begin{aligned}
\operatorname{diag}\left(\left[\frac{\widehat{\mu}_{L}}{\widehat{\mu}_{L-1}}, \ldots, \frac{\widehat{\mu}_{0}}{\widehat{\mu}_{1}}\right]\right) & =\boldsymbol{\Gamma}_{J 2} \boldsymbol{\Gamma}_{J 1}^{-1} \operatorname{diag}\left(\widehat{\mathbf{d}}_{\mathrm{opt}}\right), \\
\widehat{\mathbf{c}}^{\prime} & =\frac{\left(\widehat{\boldsymbol{\Theta}}^{-1} \widehat{\boldsymbol{\mu}}^{\prime}\right)}{\left(\widehat{\boldsymbol{\Theta}}^{-1} \widehat{\boldsymbol{\mu}}^{\prime}\right)_{1}}
\end{aligned}
$$

where $\widehat{\boldsymbol{c}}^{\prime}=\left[1, \widehat{\boldsymbol{c}}_{1}, \ldots, \widehat{\boldsymbol{c}}_{L}\right]^{T}, \widehat{\boldsymbol{\mu}}^{\prime}=\left[\widehat{\mu}_{0}, \widehat{\mu}_{1}, \ldots, \widehat{\mu}_{L}\right]^{T}$, and $\widehat{\boldsymbol{\Theta}}=$ $\Theta(1: L+1,1: L+1)$, which means that $\widehat{\Theta}$ consists of the first $L+1$ rows and $L+1$ columns of $\Theta$ (see (15)). In (40), $\widehat{\mu}_{0}$ is a nonzero parameter, but it will be cleared by the normalization operation.

Method 1 can only estimate $L$ mutual coupling parameters. 
Method 2. In addition, we can obtain a more accurate estimate of $\mathbf{c}$ by the method introduced in [7]. Consider

$$
\begin{aligned}
& \widehat{\mathbf{c}}^{\prime}=\underset{\mathbf{c}}{\arg \min } \mathbf{c}^{\prime H} \mathbf{\Omega} \mathbf{c}^{\prime} \\
& \text { s.t. }\left(\widehat{\mathbf{c}}^{\prime}\right)_{1}=c_{0}=1 \\
& \mathbf{\Omega}=\mathbf{\Upsilon}^{H}(\mathbf{a}(\widehat{\theta})) \widehat{\mathbf{U}}_{n} \widehat{\mathbf{U}}_{n}^{H} \mathbf{Y}(\mathbf{a}(\widehat{\theta})),
\end{aligned}
$$

where $\widehat{\mathbf{c}}^{\prime}=\left[1, \widehat{c}_{1}, \ldots, \widehat{c}_{N}\right]^{T}$. If $\boldsymbol{\phi}_{\min }$ is the eigenvector of $\boldsymbol{\Omega}$ corresponding to its minimum eigenvalue, then

$$
\widehat{\mathbf{c}}^{\prime}=\frac{\phi_{\min }}{\left(\phi_{\min }\right)_{1}}
$$

We give a simpler expression of $\Upsilon(\mathbf{a}(\widehat{\theta})) \in C^{M \times(N+1)}$ than that in $[7,9]$. Consider

$$
\begin{aligned}
& \Upsilon(:, 1)=\mathbf{a}(\widehat{\theta}), \\
& \Upsilon(:, j)=\operatorname{circshift}(\mathbf{a}(\widehat{\theta}), j-1)+\operatorname{circshift}(\mathbf{a}(\widehat{\theta}), 1-j), \\
& \qquad \begin{array}{l}
j=2,3, \ldots, N, \\
\Upsilon(:, N+1)
\end{array} \\
& = \begin{cases}\operatorname{circshift}(\mathbf{a}(\widehat{\theta}), N), & \text { for even } N \\
\operatorname{circshift}(\mathbf{a}(\hat{\theta}), N)+\operatorname{circshift}(\mathbf{a}(\widehat{\theta}),-N), & \text { for odd } N,\end{cases}
\end{aligned}
$$

where $\operatorname{circshift}(\mathbf{a}(\widehat{\theta}), j)$ is a function that shifts $\mathbf{a}(\widehat{\theta})$ by $j$ times circularly. If $j<0$, then it shifts $\mathbf{a}(\widehat{\theta})$ in the counter direction.

\section{Simulations}

Consider a 16-element half-wave dipole antenna UCA with $f_{0}=1.032 \mathrm{GHz}$ and $r=0.7 \lambda$. Set $L=4$. The mutual coupling vector $\mathbf{c}$ is listed in Table 1 . The theoretical parameters are calculated according to Gupta and Ksienski's formulation [6], and small values are treated as $c_{j}=0, j=3, \ldots, 8$. Real parameters should be estimated by array calibration method.

Two signals are impinging from $35^{\circ}$ and $45^{\circ}$ with the same signal noise ratio (SNR). We apply the classic MUSIC, blind RARE [9], blind R-RARE [10], and UCA-RARE [8] methods to obtain azimuth estimates. The results are shown in Figure 2.

It shows that the MUSIC method and RARE-based blind methods cannot differentiate these two signals because RARE-based methods will introduce spurious estimates. We should obtain the initial estimates from the MUSIC spectrum to start the iterative method [7], but it is difficult to find two different spectral peaks from the spectrum of "MUSIC without MCM."

We apply the proposed blind method to the above example. The estimated average DOA absolute bias and root mean square error (RMSE) versus SNR are illustrated in Figures 3-6 ( $K=10000,100$ trials). Method in [7] $(\epsilon=0.01)$ and the Cramér-Rao bound (CRB) with a known MCM are also presented [16].
TABLE 1: Mutual coupling parameters.

\begin{tabular}{cccc}
\hline$c_{0}$ & $c_{1}$ & $c_{2}$ & $c_{j}, j=3, \ldots, 8$ \\
\hline 1 & $-0.3204+0.1878 i$ & $0.1496+0.1153 i$ & 0 \\
\hline
\end{tabular}

TABLE 2: Mutual coupling estimates $(\mathrm{SNR}=30 \mathrm{~dB}, K=1000)$.

\begin{tabular}{lcc}
\hline & $c_{1}$ & $c_{2}$ \\
\hline True value & $-0.3204+0.1878 i$ & $0.1496+0.1153 i$ \\
Method 1 & $-0.3114+0.2053 i$ & $0.1451+0.1179 i$ \\
Method 2 & $-0.3203+0.1877 i$ & $0.1496+0.1154 i$ \\
\hline
\end{tabular}

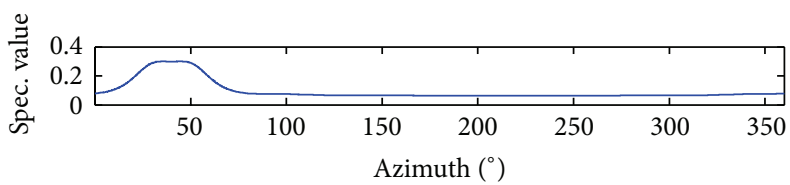

— MUSIC without MCM

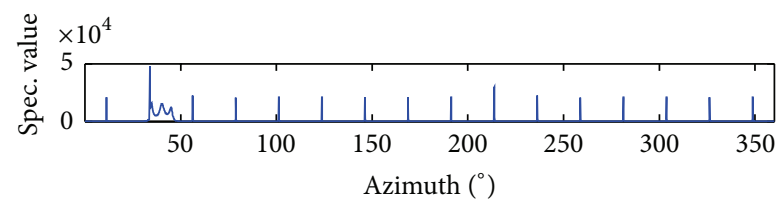

- RARE [9]

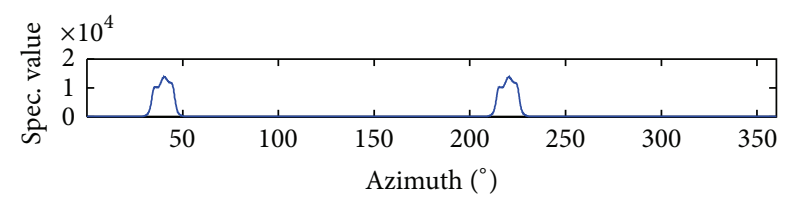

- UCA-RARE [8]

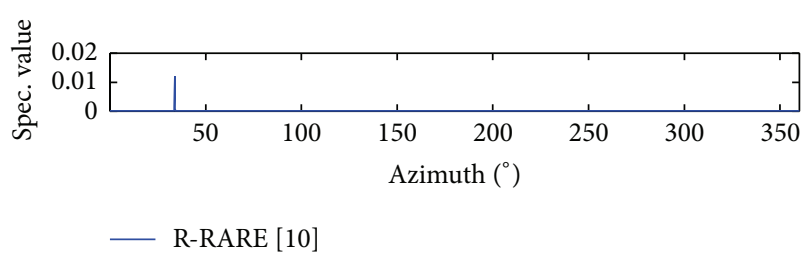

FIGURE 2: MUSIC and RARE spectrum (SNR = $20 \mathrm{~dB}, K=1000$, one trial).

This shows that all the four proposed methods can give satisfactory estimates and that the tendency of the RMSE is the same as the CRB with an increase of SNR. Method Blind-m1-full and method Blind-m2-full are more effective than the other two methods because more rows of $\widehat{\widehat{U}}_{s}$ are involved when we calculate the fitting matrix $\widehat{\boldsymbol{\Psi}}$ (see (35)). A comparison of simulations versus sampling number will give similar results. Method in [7] gives a biased estimate.

Table 2 lists the MCM parameter estimates based on the two methods introduced in Section 3.

This shows that Method 2 can give more accurate results. 


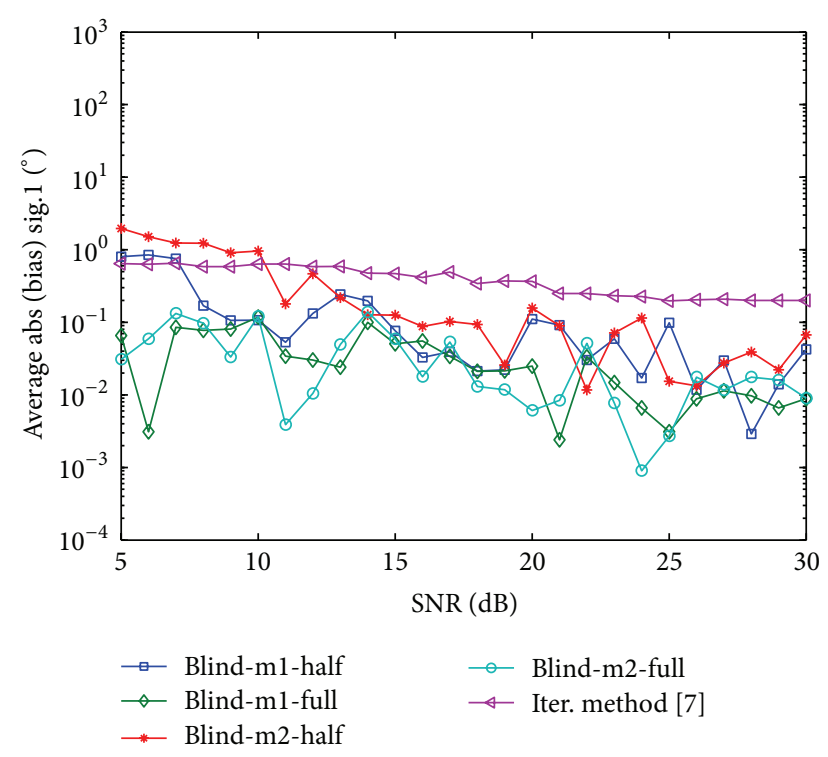

FIgURE 3: Average DOA absolute bias versus SNR for signal 1.

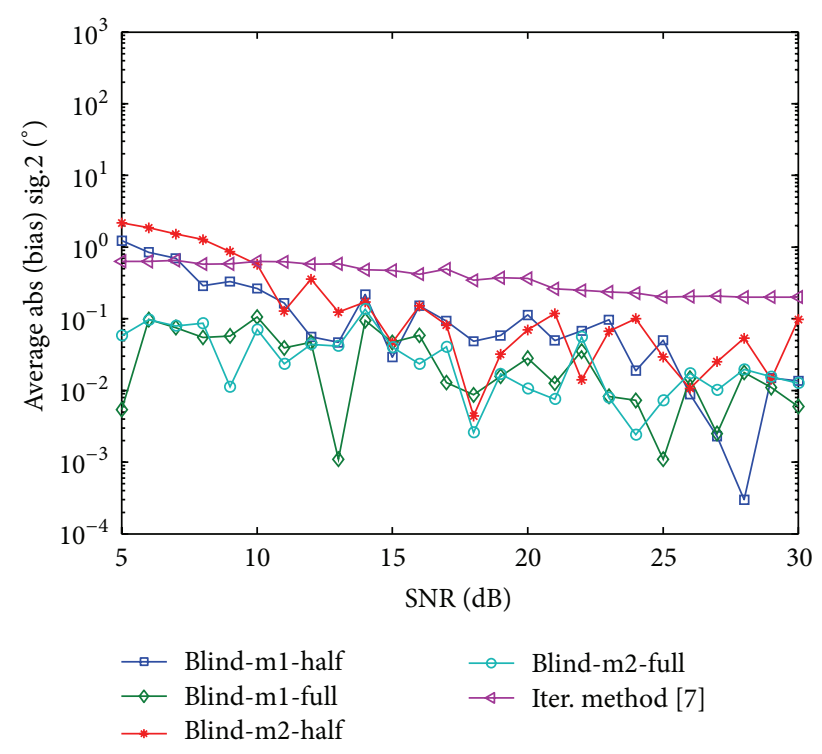

Figure 4: Average DOA absolute bias versus SNR for signal 2.

\section{Conclusion}

In DFT beam space, we utilize a modified ESPRIT algorithm to obtain a reliable DOA estimate when there is a severe mutual coupling effect. The new blind method is efficient because it avoids searching for the spectral peaks. For closely spaced signals, neither the classic MUSIC nor RARE-based methods provide a good estimate, while the proposed new method can produce an accurate estimate. Moreover, these direction estimates can be used for further MCM parameter estimation.

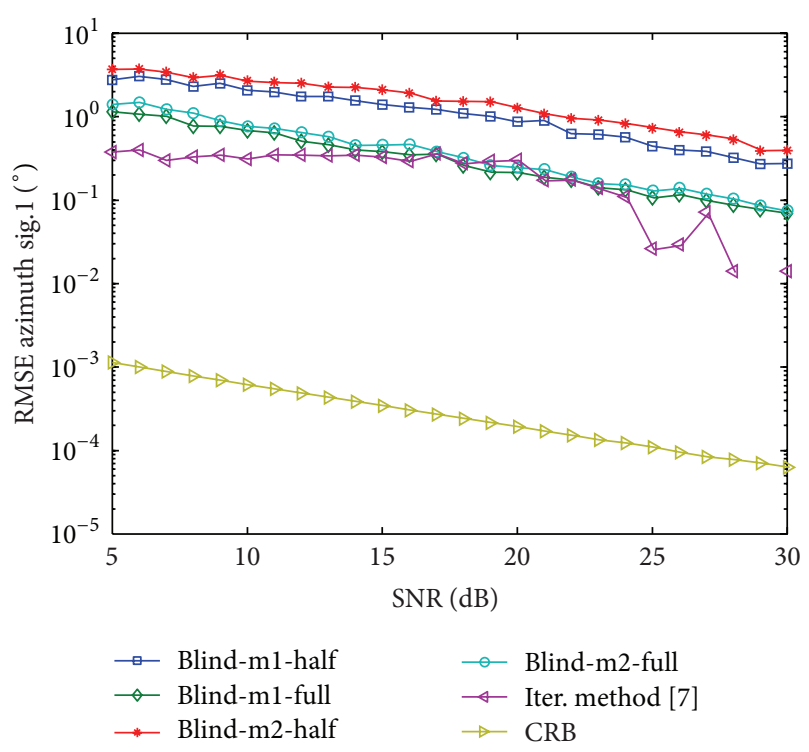

FIGURE 5: RMSE versus SNR for signal 1.

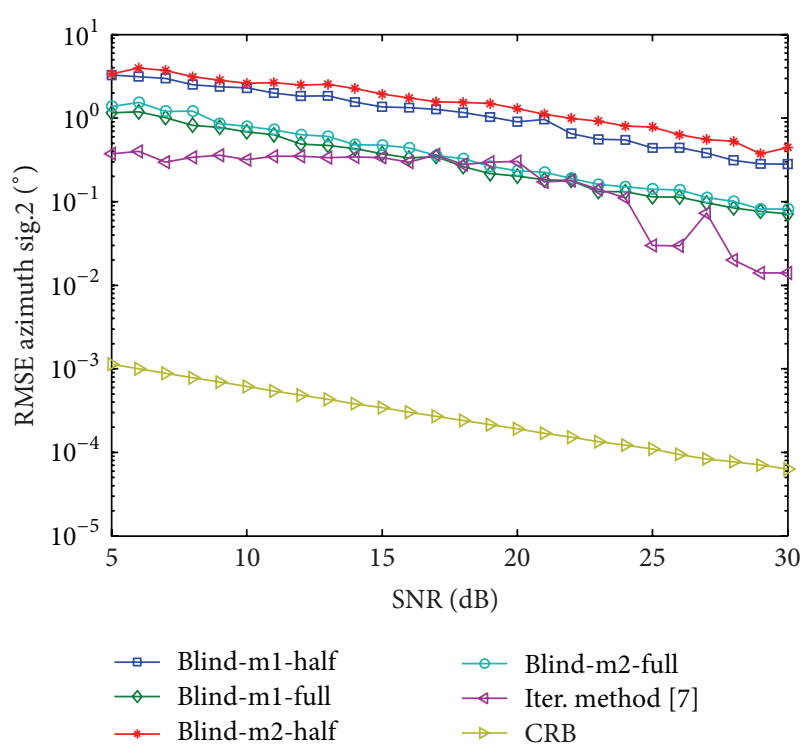

FIGURE 6: RMSE versus SNR for signal 2.

\section{Conflict of Interests}

The authors declare that there is no conflict of interests regarding the publication of this paper.

\section{Acknowledgments}

The work is supported by the Chongqing Academician Fund under Grant cstc2014yykfys90001, by the Project of Chinese Academy of Engineering under Grant 2014-XX-05, by the National Natural Science Foundation of China under Grant 61501068, by the Project of Equipment Pre-Research of the Ministry of Education of China under Grant 62501040217, and by the Fundamental Research Funds for the Central Universities under Grant 106112013CDJZR165502. 


\section{References}

[1] J. Capon, "High-resolution frequency-wavenumber spectrum analysis," Proceedings of the IEEE, vol. 57, no. 8, pp. 1408-1418, 1969.

[2] R. O. Schmidt, "Multiple emitter location and signal parameter estimation," IEEE Transactions on Antennas and Propagation, vol. 34, no. 3, pp. 276-280, 1986.

[3] R. Roy and T. Kailath, "ESPRIT-estimation of signal parameters via rotational invariance techniques," IEEE Transactions on Acoustics, Speech and Signal Processing, vol. 37, no. 7, pp. 984995, 1989.

[4] C. P. Mathews and M. D. Zoltowski, "Eigenstructure techniques for 2-D angle estimation with uniform circular arrays," IEEE Transactions on Signal Processing, vol. 42, no. 9, pp. 2395-2407, 1994.

[5] F. Belloni and V. Koivunen, "Beamspace transform for UCA: error analysis and bias reduction," IEEE Transactions on Signal Processing, vol. 54, no. 8, pp. 3078-3089, 2006.

[6] I. J. Gupta and A. A. Ksienski, "Effect of mutual coupling on the performance of adaptive arrays," IEEE Transactions on Antennas and Propagation, vol. 31, no. 5, pp. 785-791, 1983.

[7] B. Friedlander and A. J. Weiss, "Direction finding in the presence of mutual coupling," IEEE Transactions on Antennas and Propagation, vol. 39, no. 3, pp. 273-284, 1991.

[8] M. Pesavento and J. F. Böhme, "Direction of arrival estimation in uniform circular arrays composed of directional elements," in Proceedings of the IEEE Sensor Array and Multichannel Signal Processing Workshop Proceedings, pp. 503-507, Rosslyn, Va, USA, August 2002.

[9] M. Lin and L. X. Yang, "Blind calibration and DOA estimation with uniform circular arrays in the presence of mutual coupling," IEEE Antennas and Wireless Propagation Letters, vol. 5, no. 1, pp. 315-318, 2006.

[10] D. Jisheng, B. Xu, H. Nan, C. Chunqi, and X. Weichao, "A recursive RARE algorithm for DOA estimation with unknown mutual coupling," IEEE Antennas and Wireless Propagation Letters, vol. 13, pp. 1593-1596, 2014.

[11] Y. Ma, Y. Yang, Z. He, K. Yang, C. Sun, and Y. Wang, "Theoretical and practical solutions for high-order superdirectivity of circular sensor arrays," IEEE Transactions on Industrial Electronics, vol. 60, no. 1, pp. 203-209, 2013.

[12] R. H. F. Chan and X.-Q. Jin, An Introduction to Iterative Toeplitz Solvers, SIAM, Philadelphia, Pa, USA, 2007.

[13] S. Liu, L. Yang, and S. Yang, "Robust joint calibration of mutual coupling and channel gain/phase inconsistency for uniform circular array," IEEE Antennas and Wireless Propagation Letters, 2015.

[14] C. M. S. See, "Sensor array calibration in the presence of mutual coupling and unknown sensor gains and phases," Electronics Letters, vol. 30, no. 5, pp. 373-374, 1994.

[15] L. Bin and L. Guisheng, "Method for array gain and phase uncertainties calibration based on ISM and ESPRIT," Journal of Systems Engineering and Electronics, vol. 20, no. 2, pp. 223-228, 2009.

[16] P. Stoica, E. G. Larsson, and A. B. Gershman, "The stochastic CRB for array processing: a textbook derivation," IEEE Signal Processing Letters, vol. 8, no. 5, pp. 148-150, 2001. 


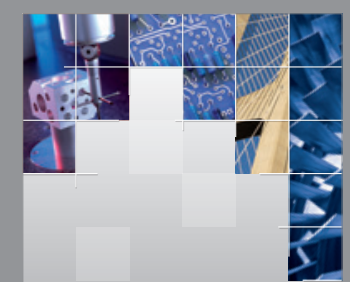

\section{Enfincering}
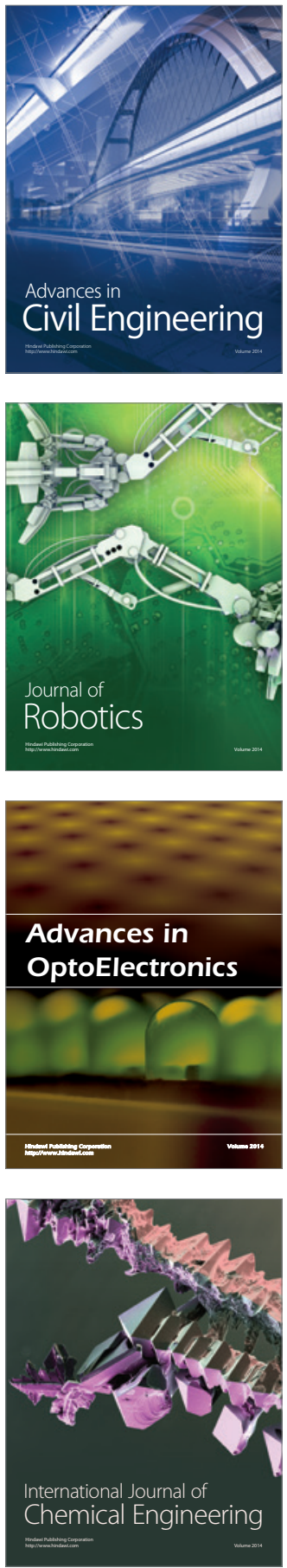

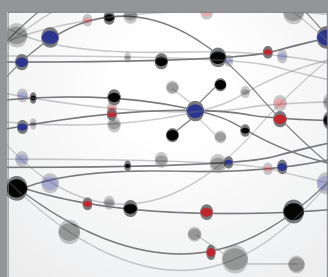

The Scientific World Journal

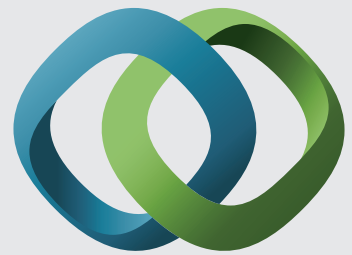

\section{Hindawi}

Submit your manuscripts at

http://www.hindawi.com
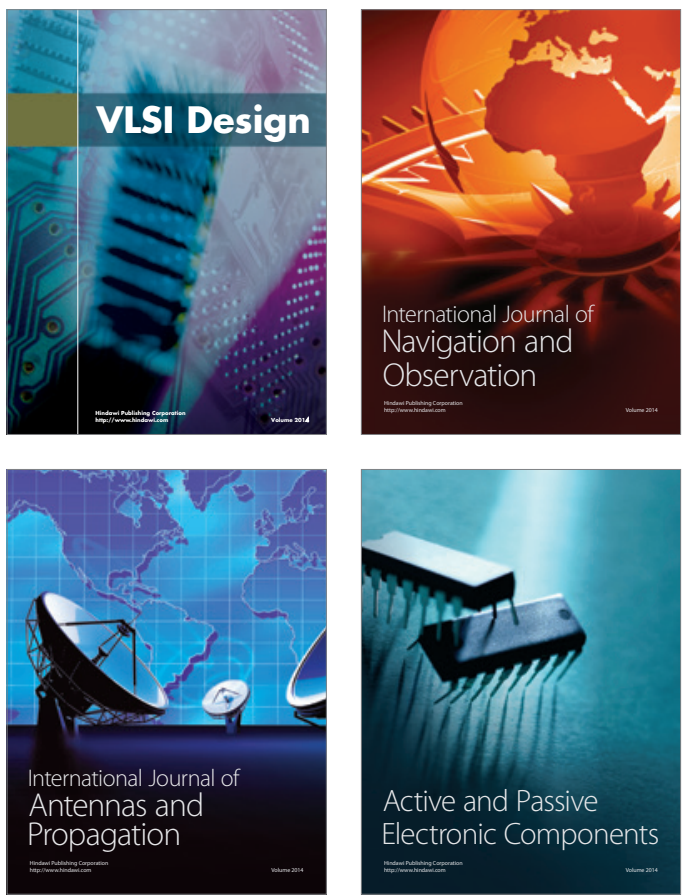
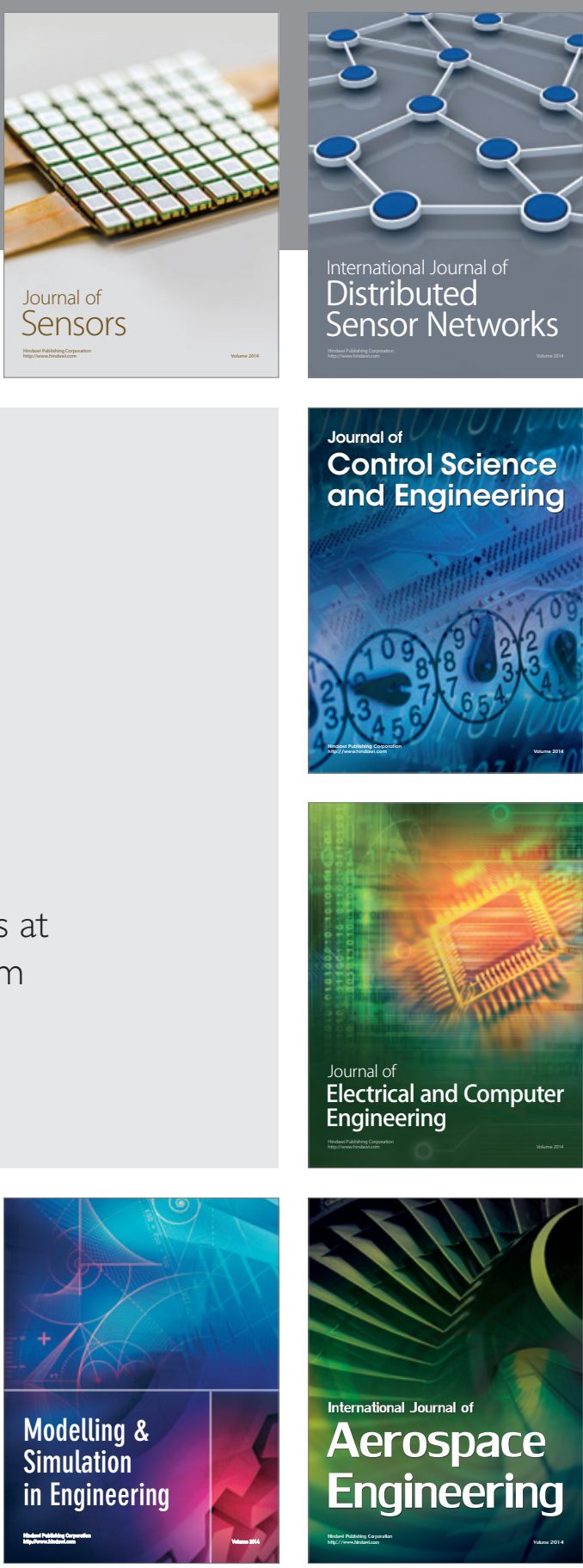

International Journal of

Distributed

Sensor Networks

Journal of

Control Science

and Engineering
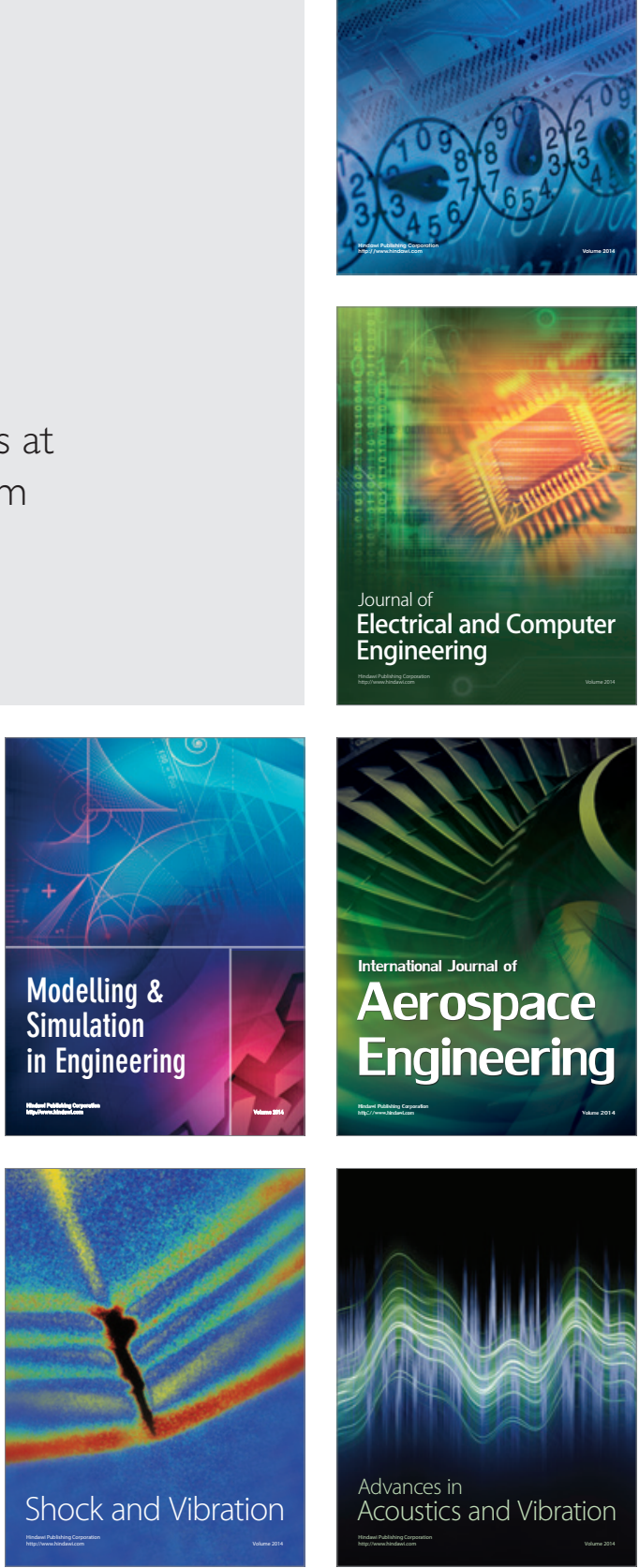